\title{
Liquid backmixing in oscillatory flow through a periodically constricted meso-tube
}

\author{
N. Reis, A.A. Vicente*, J.A. Teixeira \\ IBB-Institute for Biotechnology and Bioengineering, Department of Biological Engineering, Universidade do Minho, Campus de Gualtar, \\ 4710-057 Braga, Portugal
}

\section{A R T I C L E I N F O}

\section{Article history:}

Received 10 August 2009

Received in revised form 17 January 2010

Accepted 25 January 2010

Available online 1 February 2010

\section{Keywords:}

Backmixing

Smooth-periodic constrictions

Oscillatory flow

Meso-tube

Liquid flow

Continuous

\begin{abstract}
A B S T R A C T
This paper deals with the measurement and modelling of axial liquid dispersion in a $4.5 \mathrm{~mm}$ internal diameter tube provided with smooth-periodic constrictions (meso-tube) in steady and oscillatory flow conditions. The residence time distribution (RTD) in the meso-tube was monitored for a range of fluid oscillation frequency $(f)$ and amplitude $\left(x_{0}\right)$ at laminar flow. The RTD response was modelled with three hydrodynamic models: (i) tanks-in-series, (ii) tanks-in-series with backflow and (iii) plug flow with axial dispersion. The steady flow through the meso-tube at flow rates up to $21.30 \mathrm{ml} / \mathrm{min}$ resulted in broad RTDs, mainly due to the parabolic velocity profile. The use of fluid oscillations allowed a fine-control of the axial liquid dispersion in the meso-tube due to generation of secondary flow in the regions between the constrictions. The axial dispersion coefficient $D$ was reduced by up to 13 -fold in comparison with the steady flow situation. Values of $x_{0} \leq 1 \mathrm{~mm}$ and $f=10 \mathrm{~Hz}$ generally resulted in a maximum reduction in axial dispersion through, therefore maximum improvements in RTD. The tanks-in-series model was generally not capable of predicting RTDs in the meso-tube. The potential of this platform for the continuous, sustainable production of added-value products is herein demonstrated.
\end{abstract}

(c) 2010 Elsevier B.V. All rights reserved.

\section{Introduction}

A number of meso-technologies have recently been developed in response to a paradigm-shift in chemical and biochemical plant design. They aim at supporting the design of sustainable plants and delivering consistent quality for added-value products by operating in continuous. The last is relevant to the kilogram-per-day production of added-value chemicals and (bio)pharmaceutical products. Here one can include a novel scaled-down platform based on a $4.5 \mathrm{~mm}$ i.d. constricted tube (herein designated meso-tube) running on oscillatory flow mixing and capable of handling multiphase flow processing (gas, liquid and solid) [1]. We have recently shown the enhanced mass transfer rates delivery by the meso-tube in multiphase flow [2], further demonstrated by successful applications to the upstream intensification of bioprocesses [3]. The mixing and residence times were also briefly studied as a function of the fluid oscillation conditions (frequency and centre-to-peak amplitude) in the meso-tube [4,5]. For example, we have was shown that oscillatory flow can positively decrease axial dispersion in the meso-tube, being possible to approach the residence time distribution of a perfect plug flow behaviour [5] We aim in this study

\footnotetext{
* Corresponding author. Tel.: +351253 604419; fax: +351253678986.

E-mail address: avicente@deb.uminho.pt (A.A. Vicente).
}

at giving a more detailed insight on the modelling of residence time distributions (RTDs) in the meso-tube using different hydrodynamic models and numerical techniques, and making some considerations in relation to the high conversion and selectivity that can be produced with the new platform, with enormous potential in the intensification of upstream and downstream processing units.

Several studies have shown the general potential of curved geometries [see for example 6, 7] and oscillatory flow reactors [e.g. 16] to the process industry, in particular by demonstrating the control of RTDs through the use of a complex tube geometry.

The tracer injection and response method has been widely used to study mixing characteristics of reactors through RTD [8]. Techniques such as the moment [9], weighted moment [10], Laplace and Fourier transform domain analysis [11] and time-domain analysis $[12,13]$ have been employed to analyse the tracer response data. A good discussion of advantages and disadvantages of each technique may be found elsewhere [14]. Time-domain analysis of RTD in baffled tubes such as conventional (50-mm-i.d.) oscillatory flow reactors (OFRs) has been reported by several authors [15-19] using conventional techniques, e.g. conductivity measurement. A major issue with such technique is the effect of the tracer density (solution of $\mathrm{NaCl}$ or $\mathrm{KNO}_{2}$ ) which affects the sensitivity of the determination. More recently a non-intrusive state-of-the-art laser-induced fluorescence technique [20] was applied (using a dye that 
fluoresces when induced by a laser) to study the RTD in conventional OFRs, being concluded that intrusive experiments using conductivity probes can have mass transfer associated problems into the membranes of the probes, originating misleading results. In most of the studies available, two different models were used to determine dispersion coefficients: (1) plug flow with axial dispersion and (2) series of CSTRs with backflow. Similar results were achieved with these two models.

Briens et al. [21] demonstrated that significant differences can exist between the real RTD curve and a concentration profile measured by a "through-the-wall" technique (instead of "mixed cup" concentrations) when flow behaviour is far from plug flow. This usually leads to errors in the determination of liquid residence times by the tracer injection technique. Thus, a correct specification of the system boundaries is paramount on determining the axial dispersion coefficients.

The dye injection technique and optical fibre micro-probes were herein used for monitoring online the RTDs in the meso-tube at different laminar fluid flow rates and several combinations of fluid oscillation frequency and centre-to-peak amplitude. The RTDs were then modelled using different techniques (moments fitting, timedomain curve fitting and Laplace-domain curve fitting) to a set of single-parameter hydrodynamic models considering both perfect and imperfect injection pulse. Later on, response curves were used to estimate the degree of deviation from an ideal plug flow for a first-order homogenous, isothermal, chemical reaction conversion in the meso-tube.

\section{Experimental}

\subsection{Setup and materials}

RTD experiments were performed with a dye tracer in a $350 \mathrm{~mm}$ long glass tube using imperfect pulse response as illustrated in Fig. 1. The glass tube has an internal diameter of $\sim 4.5 \mathrm{~mm}$ and presents smooth-periodic constrictions along its length. The average distance between adjacent constrictions $(l)$ is $\sim 13 \mathrm{~mm}$, therefore the ratio $l / d \cong 3$, which is significantly higher than the ratio $l / d=1.5$ found for conventional OFRs. This gives a cross-free section area $\left(\alpha=d_{0}^{2} / d\right)$ equal to $87 \%$, considering the internal diameter of the tube in the constrictions $\left(d_{0}\right)$ is $\sim 1.6 \mathrm{~mm}$. The mean (cross-section weighted) tube-internal diameter $(\bar{d})$ is $\sim 4.0 \mathrm{~mm}$.

A single tube was fixed vertically as shown in Fig. 1. A steady, fluid flow rate ( $v=1.67-21.3 \mathrm{ml} / \mathrm{min}$ ) was continuously delivered by a peristaltic pump and passed through a reservoir in order to eliminate fluid pulsations originated by the peristaltic pump. Controlled fluid oscillations were then imposed in the continuous net flow by a rotative ceramic piston pump (CKCRHO, Fluid Metering Inc., New York), working in closed-loop. The centre-topeak amplitude $\left(x_{0}\right)$ of the fluid oscillations was controlled by the volume-per-stroke of the piston volume in the pump head in the range of $0-3 \mathrm{~mm}$. The fluid oscillation frequency $(f)$ was controlled by the setting the angular rotation speed of the motor. The range of $f=0-20 \mathrm{~Hz}$ was tested in this work.

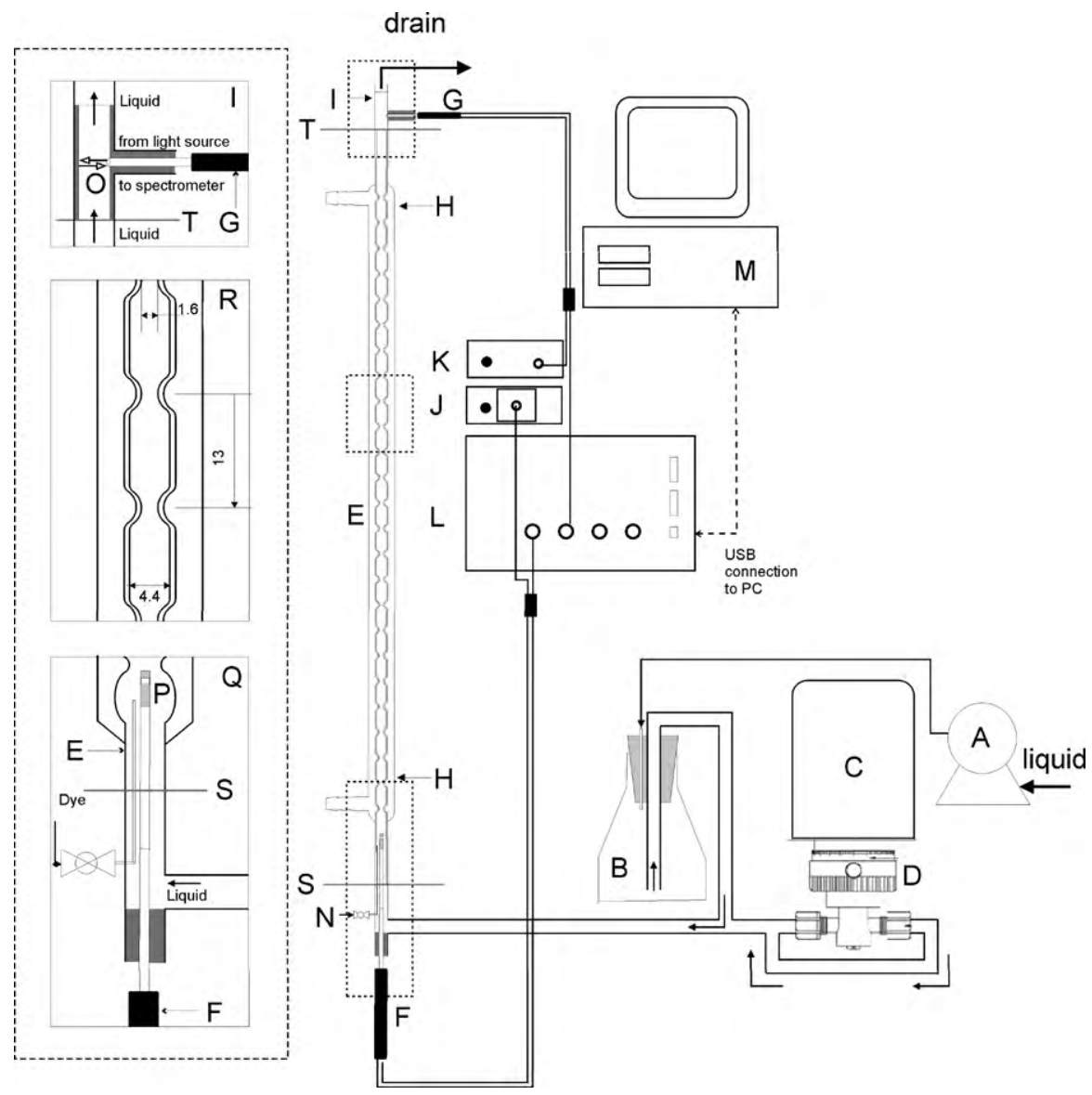

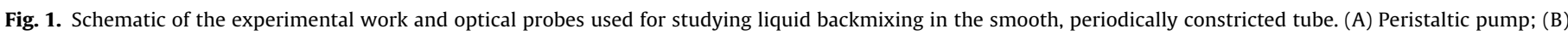

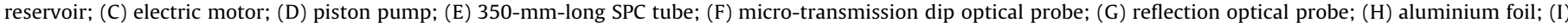

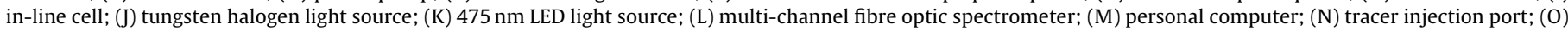

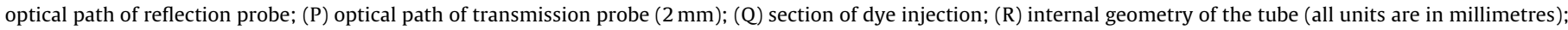
(S) inlet detail; (T) outlet detail. 


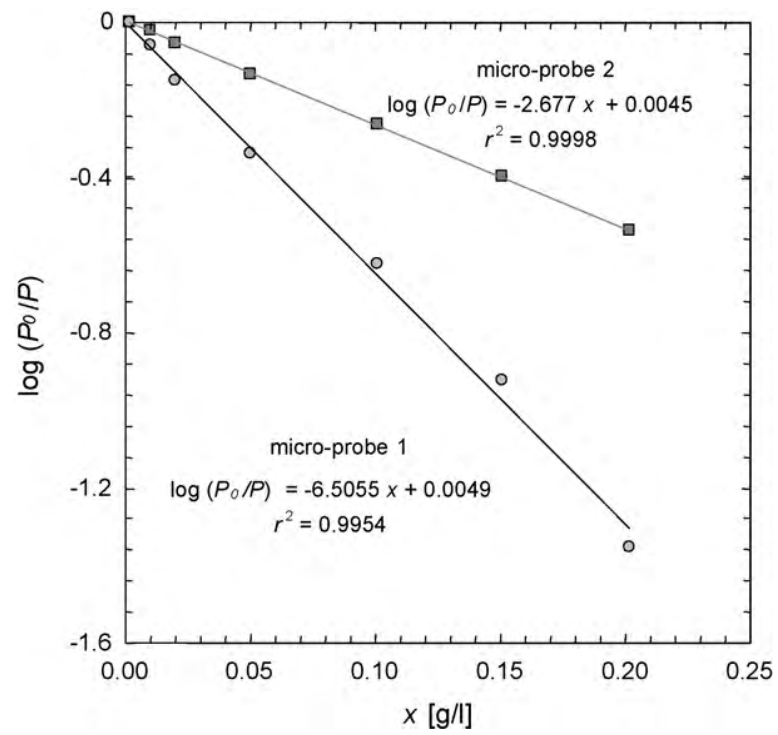

Fig. 2. Calibration curves for micro-probes in terms of absorbance, $A b s=\log \left(P_{0} / P\right)$, for increasing tracer concentrations $(x)$.

An aqueous solution of indigo carmine (Merck, Darmstadt, Germany) was used as the dye tracer for the RTD experiments and presents an absorbance peak at $610-612 \mathrm{~nm}$. It was selected for not adsorbing to the installation pipes or the tube walls.

The optical density (OD) of liquid flow was measured in two point of the tube via optical micro-probes connected to a multi-channel optic spectrometer system (Avantes, Eerbeek, The Netherlands). A micro-transmission dip optical probe (micro-probe 1) (FDP-UV-micro-1), with $2 \mathrm{~mm}$ optical length, was used to monitor the (local) tracer concentration inside the first cavity of the tube (at an axial distance of $15 \mathrm{~mm}$ from the inlet). The outlet concentration (at an axial distance of $358 \mathrm{~mm}$ from the inlet point) was measured with a reflection probe (micro-probe 2) (FCR-7UV200-1.5×100-2, $1.5 \mathrm{~mm}$ diameter tip) installed perpendicularly to the flow direction as detailed in Fig. 1. This probe was installed in a $5 \mathrm{~mm}$ internal diameter in-line flow cell having a reflecting-white background, therefore interrogating the cross averaged (through-the-wall) concentration. The whole setup was covered with an aluminium foil to reduce noise from the environment light.

An integral function $P=\int_{585 \mathrm{~nm}}^{635 \mathrm{~nm}} I(\lambda) d \lambda$ (where $\lambda$ is the wavelength and $I(\lambda)$ is the intensity of spectrum) were defined in the Avasoft (Avantes, Eerbeek, The Netherlands) allowing monitoring the OD with each probe using a pre-optimised integration time in the $\lambda$ range $585-635 \mathrm{~nm}$. Light spectrum were averaged three times (for more stable values), resulting on values of $P$ every $1 / 3 \mathrm{rd}$ of a second. The OD or liquid absorbance defined as $A b s=\log \left(P_{0} / P\right)$ was highly linear for both micro-probes for values of $x \leq 0.2 \mathrm{~g} / \mathrm{l}$, as seen in Fig. $2\left(P_{0}\right.$ is the reference incident light intensity for water).

A major property of oscillatory flow mixing is that secondary flow (i.e. flow reversing) occurs only in the vicinity of tube constrictions. As a consequence, the fluid backmixing generated by the oscillatory movement of the fluid in the plain sections of the tube should be negligible. Therefore, the boundaries conditions were assumed of the 'closed-closed' type, allowing to obtain the true RTD in the control volume [22].

\subsection{Measurement of response curves}

After extensive washing of the tube with distilled water an aqueous solution of Indigo carmine with a concentration, $x_{i n}=0.2 \mathrm{~g} / \mathrm{l}$ was continuously loaded to the tube through a special tracer injection port as seen in Fig. 3 until the readout of the probes was stable. Thereafter, the injection of the dye was stopped and the peristaltic pump and fluid oscillations started at the same time $(t=0)$, allowing a step-down response of the tracer. The step input injection method was selected as it is easier to generate experimentally when compared to the impulse technique. All experiments were performed at room temperature $\left(20^{\circ} \mathrm{C}\right)$ and using distilled water as the fluid.

A perfect pulse disturbance can be expressed $[22,23]$ by means of the cumulative distribution function evaluated from the tracer concentration at the exit $\left(x_{\text {out }}\right)$ of the tube:

$F_{t}=1-\frac{x_{\text {out }}(t)}{x_{\text {in }}} \quad F_{\theta}=1-\frac{x_{\text {out }}(\theta)}{x_{\text {in }}}$

where $\theta$ is the dimensionless residence time and is give by $t / \bar{t}$. The reduced distribution function results from derivation:

$E_{t}=\frac{d F_{t}}{d t} \quad E_{\theta}=E_{t} \bar{t}$
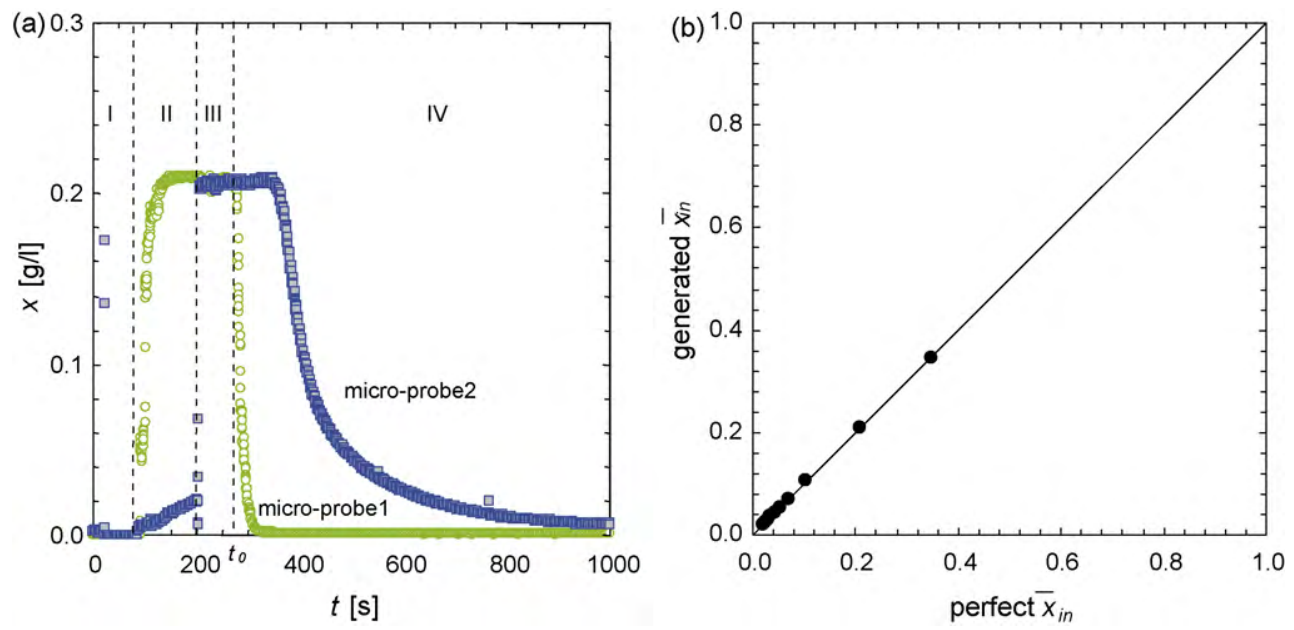

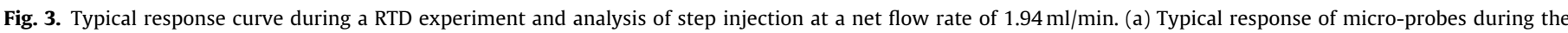

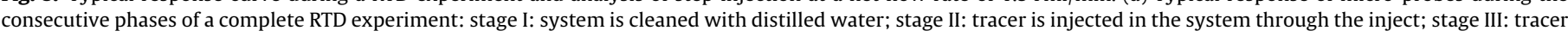

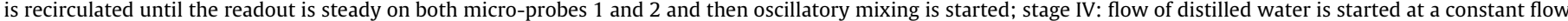

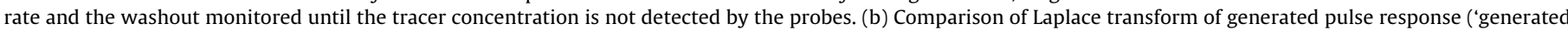

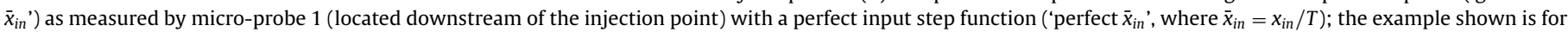
a steady, continuous flow, i.e. $\operatorname{Re}_{0}=0$ and $v=1.94 \mathrm{ml} / \mathrm{min}$. 

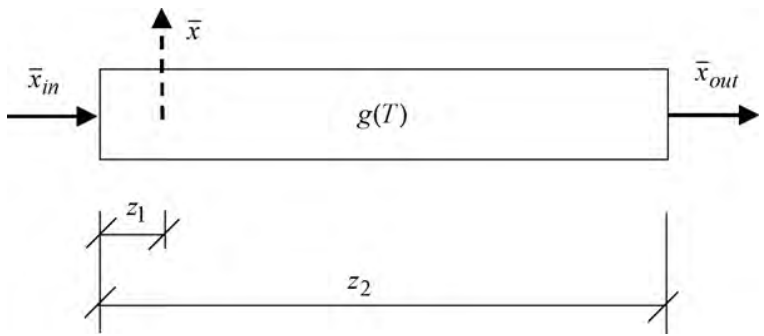

Fig. 4. Simplified representation of the transfer function, $g(T)$, of the constricted tube.

Moments are often used to characterise the response curves in terms of statistical parameters. The mean residence time $(\bar{t})$ is given by the first moment of the distribution function:

$\bar{t}=\int_{0}^{x_{\text {in }}} t \frac{1}{x_{\text {in }}} d x$

and the spread in residence time (characterised by the variance, $\sigma^{2}$ ) is given by [24]:

$\sigma^{2}=\int_{0}^{x_{i n}} t^{2} \frac{1}{x_{i n}} d x-\bar{t}^{2}$

The normalised response curves were fitted to the following three single-phase hydrodynamics models $(\mathrm{A}-\mathrm{C})$ :

A. Plug flow with axial dispersion (differential reactor [25]);

B. Series of continuous stirred tank reactors (CSTRs) without interaction [26];

C. Series of CSTRs with backflow (stagewise reactor [25]);

The model parameters being fitted were: (i) axial dispersion coefficient, $D\left(\mathrm{~m}^{2} / \mathrm{s}\right)$, (ii) number of tanks-in-series, $N_{\text {tis }}$, and (iii) backmixing coefficient, $G$ (dimensionless), respectively. Note the mean residence time of the tracer $\bar{t}$ was determined from the tracer responses, therefore $\bar{t}$ was also a parameter contributing to the model.

Model B represents the ideal behaviour of the constricted tube, at which each individual cavity in the tube would behaves as a small, independent stirred tank reactor. In this work, $N_{\text {tis }}$ was taken as 26 , which corresponds to the number of inter-constriction regions in a $350 \mathrm{~mm}$ long tube. On the other hand, models $A$ and $C$ were used for being capable of capturing the intermediate backmixing state expected for the constricted tube when operated at oscillatory flow conditions.

An analytical solution to the distribution functions correspondent to each of the three models above mentioned can be obtained by solving the mass balance equations in the Laplace domain. The time-domain solution for the axial dispersion model (A) can only be obtained for specific type of boundary conditions and measurements (open-open and through-the-wall measurements), as previously shown by Levenspiel [22]. On Laplace domain, the hydrodynamic model corresponds to a transfer function $g(T)$ as determined from a mass balance and the system response is reduced to $\bar{x}_{\text {out }}$ as illustrated in Fig. $4 ; z_{1}$ and $z_{2}$ are the dimensionless distances of probes to inlet and equal to 0.04 and 1.0 , respectively.

The analysis of the integral convolution of the tracer concentration from probe 1 (i.e. $\bar{x}$, measured at $z_{1}=0.04$ ) confirmed the perfect step input assumption for all tested values of $v$, as shown in Fig. $3 \mathrm{~b}$. The $\bar{x}_{\text {in }}$ obtained from the deconvolution of $\bar{x}$ fitted well the perfect input function given by. $\bar{x}_{i n}=C_{0} / T$ (maximum devi- ation was lower than $0.04 \%$ and the cross-correlation coefficient, $r^{2}=0.9999$ )

In situations where the response curve is not Gaussian (i.e. not symmetric in relation to the mean residence time), the exact value determined for each hydrodynamic parameter $D, N_{\text {tis }}$ or $G$ is very dependent on the numerical technique used to fit the data. Therefore, three different best-fitting methods (I-III) were used:

(I) Direct fitting of the moments of the response curves $\left(\bar{t}\right.$ and $\left.\sigma^{2}\right)$ with the moments of the function $\bar{x}_{\text {out }}$;

(II) Best-fitting of the numerical Laplace transform $g(T)$ of the hydrodynamic model with the experimental transfer function $g_{\text {out }}(T)$, given by $g_{\text {out }}(T)=\bar{x}_{\text {out }} / \bar{x}_{i n}$, where $\bar{x}_{\text {in }}=C_{0} / T$ (i.e. a perfect step input).

(III) Best-fitting of time-domain response curves $\left(F_{\theta}\right.$-diagram $)$ with the time-domain analytical solutions to the hydrodynamic models. Note that the analytical solution in time domain for hydrodynamic model $\mathrm{C}$ is limited to open-open boundary conditions and an analytical solution to model $\mathrm{B}$ in the time domain is not available.

The set of transfer functions and moment equations herein used was summarised in Table 1. Eventual advantages and disadvantages of each used best-fitting method are discussed in more detail elsewhere [14].

The objective function defined for best-fitting $F_{\theta}$-diagrams was based on least square minimisation of the difference $\Delta_{1}$ between the theoretical response $\left(F_{\theta}\right)$ and the actual, experimental response $F_{\theta}^{\text {out }}$ :

$\Delta_{1}=\int_{0}^{\infty}\left\{F_{\theta}-F_{\theta}^{\text {out }}\right\}^{2} d \theta$

and the best-fitting of the numerical Laplace transfer functions was based on the minimisation of difference $\Delta_{2}$ :

$\Delta_{2}=\int_{0.5 T_{\text {ref }}}^{2 T_{\text {ref }}}\left\{g(T)-g_{\text {out }}(T)\right\}^{2} d T$

The value of $T_{\text {ref }}$ selected was 2.0 as higher values would result in overestimating the data in the tail (where concentration measurements are normally less accurate).

\section{Results and discussion}

\subsection{Effect of net flow rate on liquid RTD for steady flow in the tube (no fluid oscillations)}

The effect of net flow rate, $v$ on axial dispersion was inspected in the absence of any fluid oscillations in a range of $v=$ 1.67-21.30 ml/min, which corresponds to superficial liquid velocities, $u_{L S}=4 v / \pi d^{2}$ in the range of $0.18-2.34 \mathrm{~cm} / \mathrm{s}$ and net flow Reynolds numbers, $R e_{n}=u_{L S} \rho d / \mu$ of 8-102 (i.e. well within the laminar flow regime). Correspondence between $v, u_{L S}$ and $R e_{n}$ is summarised in Table 2 .

Fig. 5 summarises the normalised through-the-wall distribution (i.e. $E_{\theta}$ versus $\theta$ ) for increasing $u_{L S}$ values in the meso-tube. The $E_{\theta}$ curve for pure-convective laminar flow is also presented. This type of flow is normally obtained only with very viscous fluids and can be represented by [27]:

$E(\theta)=\frac{1}{2 \theta^{3}}$

The steady flow in the tube is clearly convective at very small values of $u_{L S}$, in particular at $u_{L S}=1.8 \mathrm{~mm} / \mathrm{s}$ (i.e. $R e_{n}=8$ ). With the 


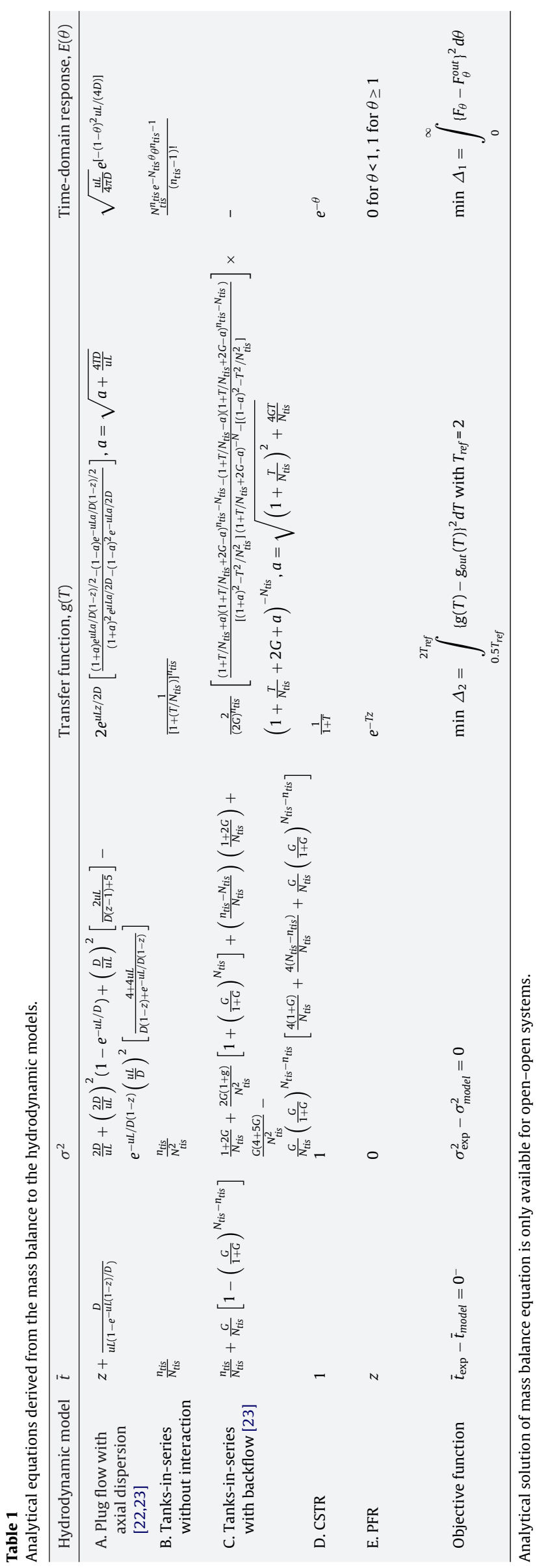

Table 2

Values of superficial liquid velocity $\left(u_{L S}\right)$, liquid flow rate $(v)$ and net flow Reynolds number $\left(R e_{n}\right)$ tested for steady flow conditions. All values are based on an internal diameter of the tube, $d=4.4 \mathrm{~mm}$.

\begin{tabular}{lllllll}
\hline$v[\mathrm{ml} / \mathrm{min}]$ & 1.67 & 1.94 & 2.98 & 5.60 & 10.83 & 21.30 \\
$u_{L S}[\mathrm{~mm} / \mathrm{s}]$ & 1.8 & 2.1 & 3.3 & 6.1 & 11.9 & 23.4 \\
Approx. $\operatorname{Re}_{n}[-]$ & 8 & 9 & 14 & 27 & 52 & 102 \\
\hline
\end{tabular}

increase in $u_{L S}$, the RTD becomes broader and the $E_{\theta}$-distribution significantly deviates from the convective laminar flow, whilst the residence time of the liquid in the tube decreases. A significant part of the fluid elements reaches the exit of the tube before $\theta<0.5$, nevertheless the fraction of fluid elements delayed for $\theta=1$ or above increases as $u_{L S}$ goes up. The generation of short-circuits through the constricted meso-tube is a problem due to the fact that most of streamlines are located at the central regions of the tube as $v$ goes higher. At the same time, extended tracer tails were detected in the response curves because of "stagnant" fluid regions produced in the inter-constrictions space. This is illustrated in Fig. 6b by the streamline plots predicted by the $2 \mathrm{D}$ axisymmetric numerical simulations in the tube using Fluent software (further details for these simulations can be found in Reis et al. [1]). Such broadening in the concentration response can be partially captured by the axial dispersion model as an increase in the "axial dispersion"; however, a good matching between the response curves at laminar flow and the axial dispersion model was not possible to obtain as that model assumes no radial concentration profile across the tube.

The broad RTDs of the type shown above are representative of the flow through tubular systems: the increase of $u_{L S}$ results in lower mean hydraulic times in the tube, therefore relative the variance increases. This is normally overcome in chemical processing plants by operating the tubular systems in the turbulent flow regime, at the expense of high-energy dissipation and long tubes required to attain significant residence times.

\subsection{Control of RTD in the tube using oscillatory flow mixing}

In this section it is shown how the liquid backmixing can be controlled in the constricted meso-tube and sharper RTDs produced simply by imposing a periodic, sinusoidal oscillation in the fluid. Several experiments were carried out at increasing values of $f$ and $x_{0}$ in the range of $0-20 \mathrm{~Hz}$ and $0-3 \mathrm{~mm}$, respectively, using a

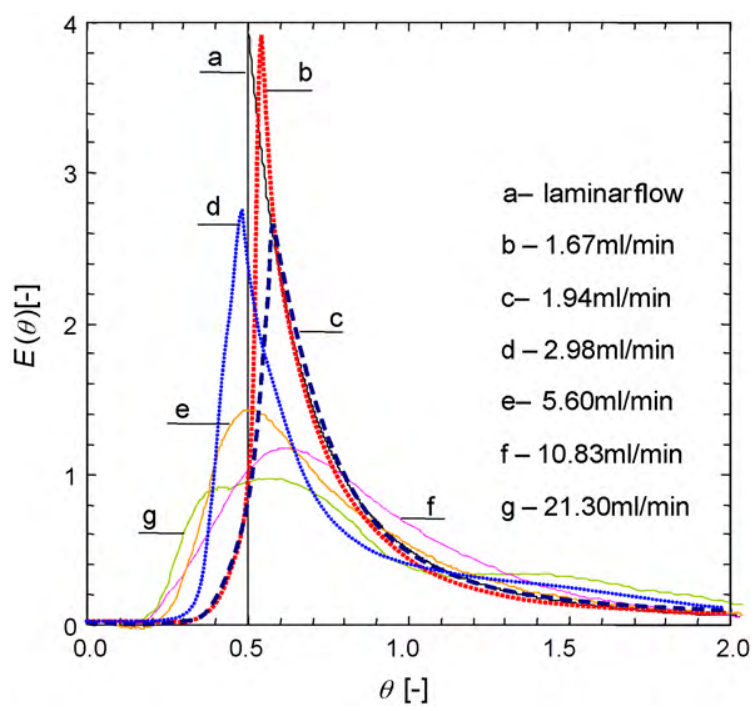

Fig. 5. Normalised tracer concentration at the outlet of the constricted tube for increasing steady flow rates, $v$; response curve for a pure-convective (laminar) flow [27] is also shown (curve a). 
(a)

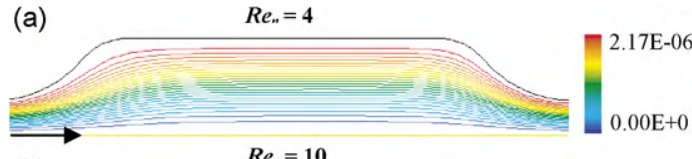

(b)

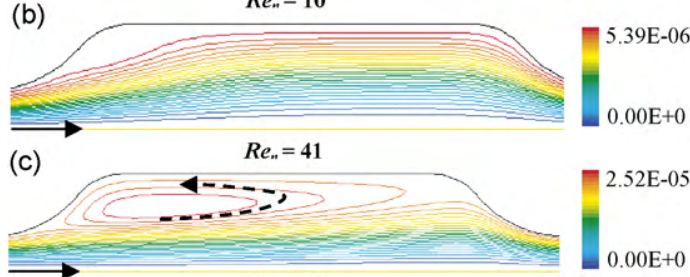

(d)
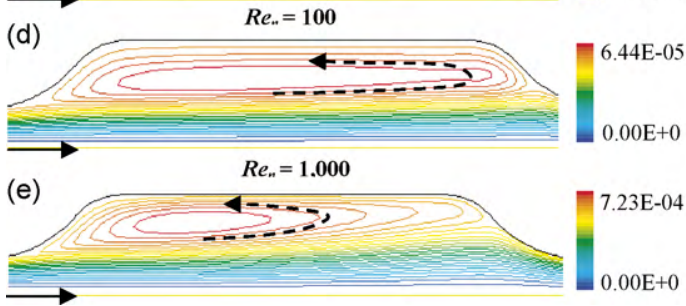

(f)

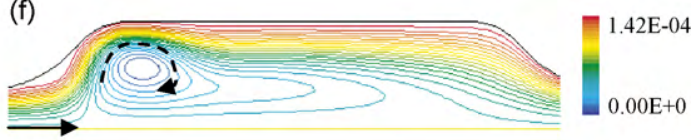

(g)
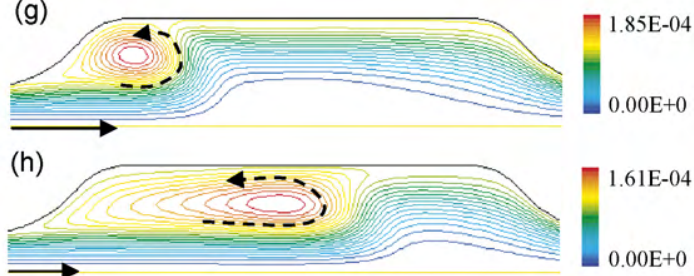

(i)
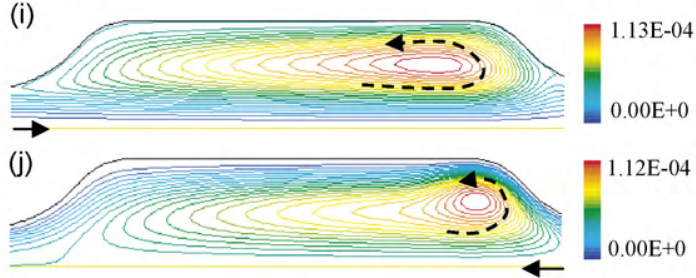

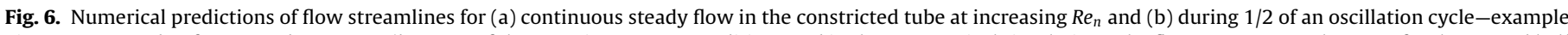

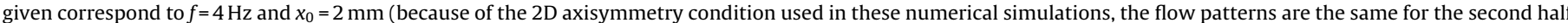

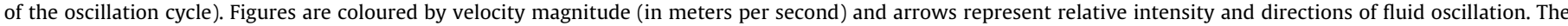

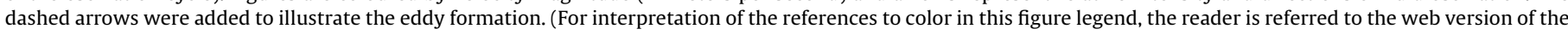
article.)

fixed value of $v=1.94 \mathrm{ml} / \mathrm{min}$ (i.e. $R e_{n} \cong 9$ ). All experiments were performed at least in duplicate with the response curves highly reproducible as shown in Fig. $7 \mathrm{a}$. The minimum value selected for the oscillatory Reynolds number, $R e_{0}=2 \pi f x_{0} \rho d / \mu$ (where $f$ is in $\mathrm{Hz}$ and $x_{0}$ in $\mathrm{m}$ ) was $R e_{0}=41$, corresponding to $f=3 \mathrm{~Hz}$ and $x_{0}=0.5 \mathrm{~mm}$. This gave a ratio $R e_{0} / R e_{n}>4$, therefore keeping the contribution of the steady net flow to backmixing insignificant in comparison to the flow patterns generated by the oscillatory flow mixing nature.

Fig. 7b summaries the $C(t)$-curves as monitored at the outlet of the constricted meso-tube at for increasing values of $x_{0}$ and a constant $f=20 \mathrm{~Hz}$. The RTD was made clearly sharper with the introduction of oscillatory flow mixing. This relates to the formation and detachment of vortex rings from the surface of the tube constrictions, assuring a high level of radial mixing. This is supported by the numerical simulations above shown in Fig. $6 \mathrm{~b}$ and further details can be found in our previous publication [1].

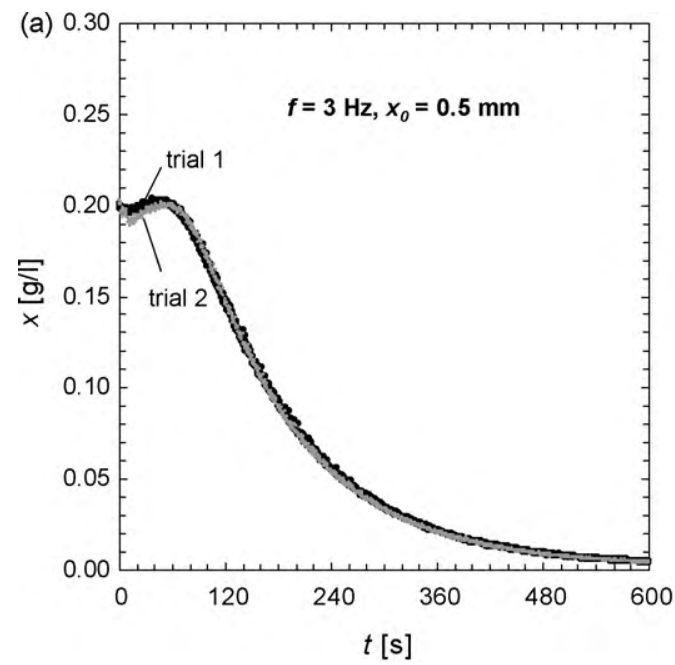

The mean value of $\bar{t}$ experimentally found in the experiments at $v$ were summarised in Fig. 8, and was concluded that $\bar{t}$ remains approximately constant (mean $\bar{t}=: 200 \pm 7 \mathrm{~s}$ ) throughout the set of conditions. This ultimately means that the numerical fitting to the hydrodynamic models A-C herein tested was simplified to a single-parameter fitting problem.

Fig. 9 shows the normalised, cumulative tracer concentration ( $F_{\theta}$-diagrams) as measured by the two probes for two experiments performed at two extreme values of $R_{0}$ : 41 and 1629. Micro-probe 1 was located internally by the inlet of the tube, whereas microprobe 2 was located by the tube's outlet. The use of smooth fluid oscillation conditions (low $x_{0}$ and $f$ ) allowed producing narrow RTDs, whilst higher values of $x_{0}$ and $f$ (in this case $20 \mathrm{~Hz}$ and $3 \mathrm{~mm}$, respectively) pushed the flow behaviour towards a highly backmixed system (i.e. with all tube behaving as a near-perfect CSTR) The RTD's sharpening at the lower values of $f$ and $x_{0}$ was associated with the generation of vortex rings in the inter-constriction regions,

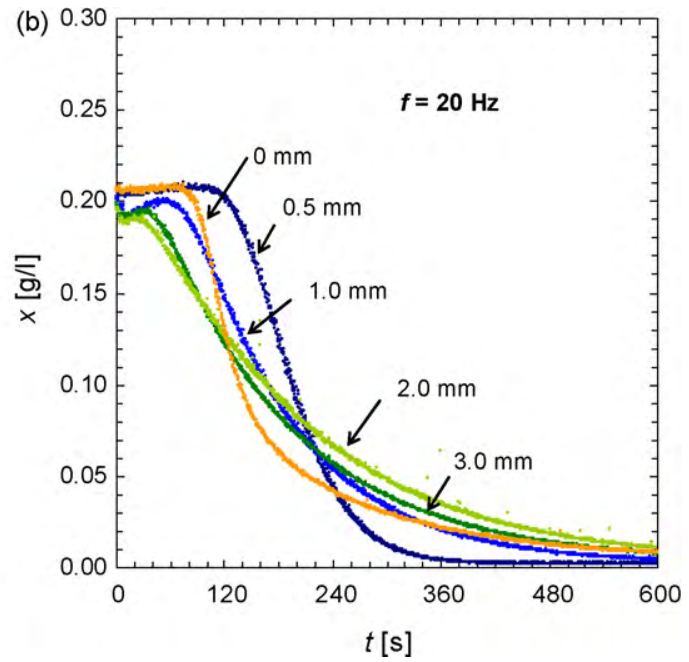

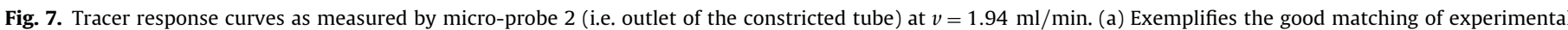
duplicates at $f=3 \mathrm{~Hz}$ and $x_{0}=0.5 \mathrm{~mm}$; (b) effect of $x_{0}$ on washout curves for a constant $f=20 \mathrm{~Hz}$ and $v=1.94 \mathrm{ml} / \mathrm{min}$. 


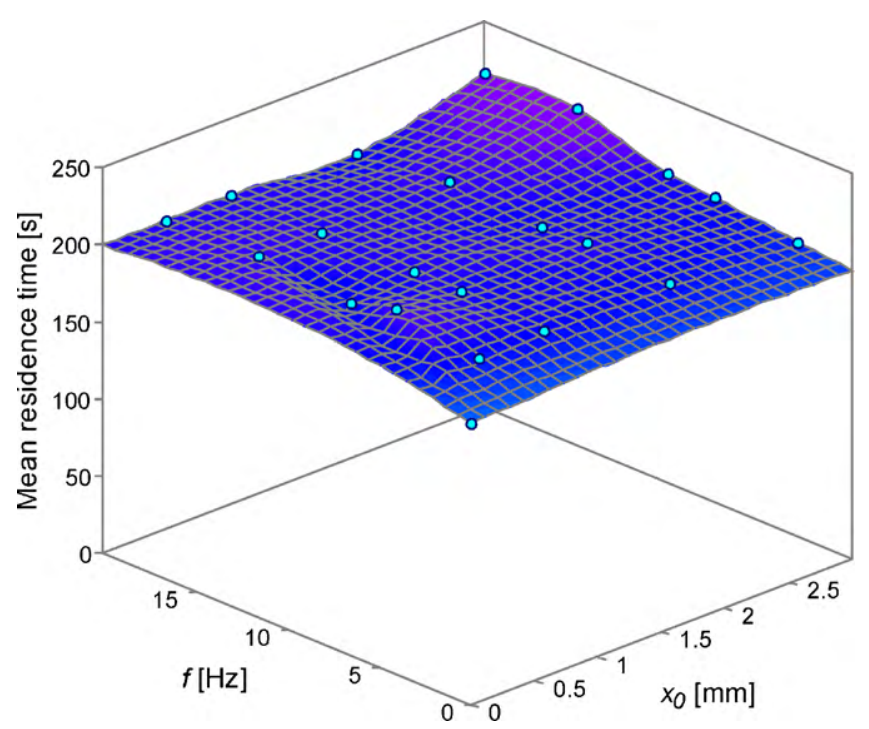

Fig. 8. Averaged, mean residence times for the tracer in the smoothly constricted tube for increasing values of $f$ and $x_{0}$ (constant $v=1.94 \mathrm{ml} / \mathrm{min}$ ).

which induced substantial radial mixing. On the other hand, the extended backflow as induced by high values of $f$ and $x_{0}$ was due to the production of convective mixing in the direction of flow [24], according to our previous detailed fluid mechanics studies carried out in the same tube geometry [1].

\subsection{Analysis of liquid backmixing in the tube and parameter fitting of hydrodynamic model}

The experimental distribution curves of the tracer were directly fitted to the response curves expected from the mass balance to each of the three hydrodynamic models tested. The best-fitting procedure was carried out using three methods as detailed above in Section 2. Fig. 10 details the mean values of axial dispersion coefficient, $D$ (associated with the plug flow model with axial dispersion, i.e. model A) in the presence of different fluid oscillation conditions and continuous $v=1.94 \mathrm{ml} / \mathrm{min}$.

The values of $D$ estimated for oscillatory flow were in the range of $1.7-33.5 \times 10^{-5} \mathrm{~m}^{2} / \mathrm{s}$ and for steady flow was $7.4-28.9 \times 10^{-5} \mathrm{~m}^{2} / \mathrm{s}$, depending on the method selected to estimate the model parameter. The use of fluid oscillations in general allowed achieving a up to a 13 -fold decrease in $D$. Small values of $x_{0}=0.5-1.0 \mathrm{~mm}$ resulted in a maximum improvement in RTDs in the tube, with estimated $D$ about one-order of magnitude lower when compared to the steady flow condition. On the other hand, values of $x_{0}=2-3 \mathrm{~mm}$ returned modest improvements in the RTD. Small values of $f$ were also found more effective on improving the RTD, whilst values of $f=15-20 \mathrm{~Hz}$ resulted in broad tracer response curves, presumably because of the highly backmixed state in the tube. The use of higher values of $f$ would represent a higher number of oscillations in the fluid for a given residence time in the tube. Smaller values of $f$ (up to $10 \mathrm{~Hz}$, inclusive) were capable of inducing the required extent of radial mixing with minimum side effect on the axial backflow between consecutive cavities. In the curves shown in Fig. 10 at constant $x_{0}$, the estimated extent of liquid backmixing was generally minimum for a value of $f=10 \mathrm{~Hz}$.

Fig. 11 summarises the two extremes values of $D$ observed on each curve at a constant $x_{0}$ Fig. 10 . It was observed a trend in minimum values of $D$ when values of $x_{0} \leq 1 \mathrm{~mm}$ were used; minimum values of $D=2.2-16.6 \times 10^{-5} \mathrm{~m}^{2} / \mathrm{s}$ were obtained for the range of $x_{0}$ herein studied. Zheng and Mackley [25] reported minimum values of $D$ in the range of $1-2 \times 10^{-5} \mathrm{~m}^{2} / \mathrm{s}$ for continuous operation of a $9 \mathrm{~m}$ long pilot oscillatory meso-reactor at the University of Cambridge, which uses the same platform herein presented. When operated with $x_{0} \approx 1 \mathrm{~mm}, f=10 \mathrm{~Hz}$ and $R e_{0}=100-300$. This is in agreement with our current experimental results.
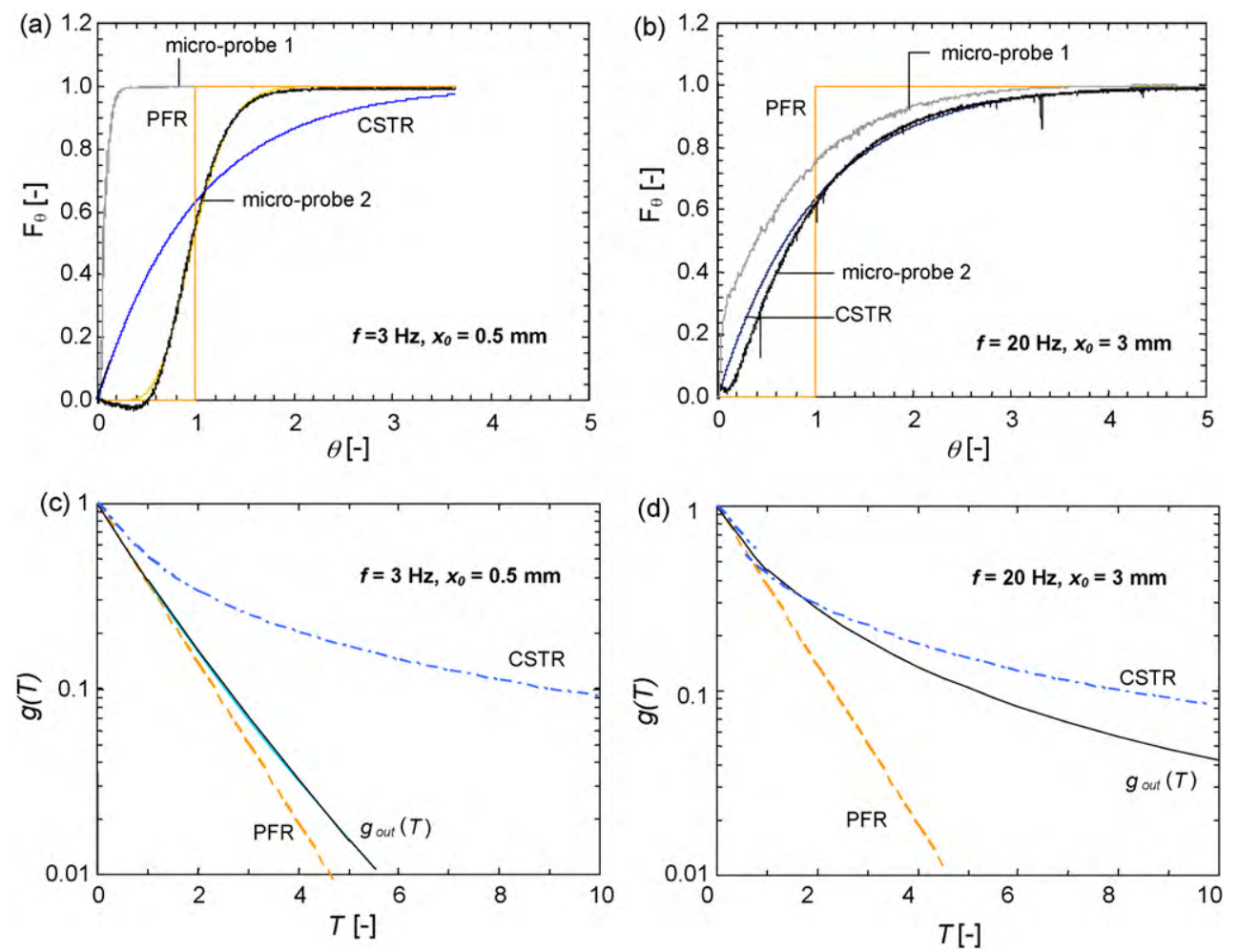

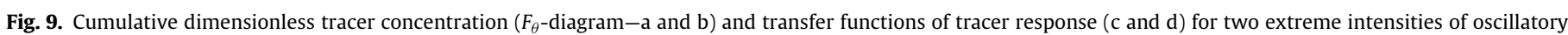
flow mixing ( $v=1.94 \mathrm{ml} / \mathrm{min})$. 

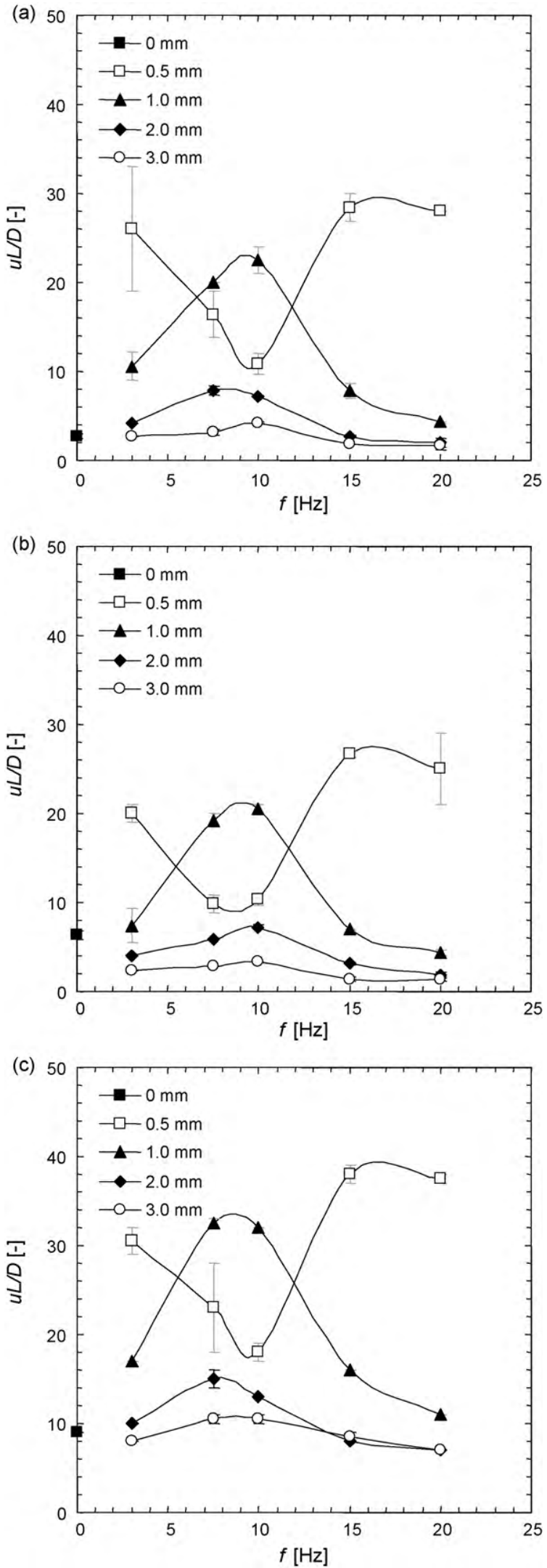

Fig. 10. Plots of the effect of $f$ and $x_{0}$ on the axial dispersion coefficient number, $u L / D$. Values of $u L / D$ where estimated by best-fitting the experimental tracer response with the plug flow model with axial dispersion with three different methods: (a) moments fitting; (b) direct fitting with the transfer function $g(T)$; (c) direct fitting with the $F_{\theta}$-diagram. Error bars represent spread ( 1 standard deviation) of $u L / D$ values for experimental duplicates.

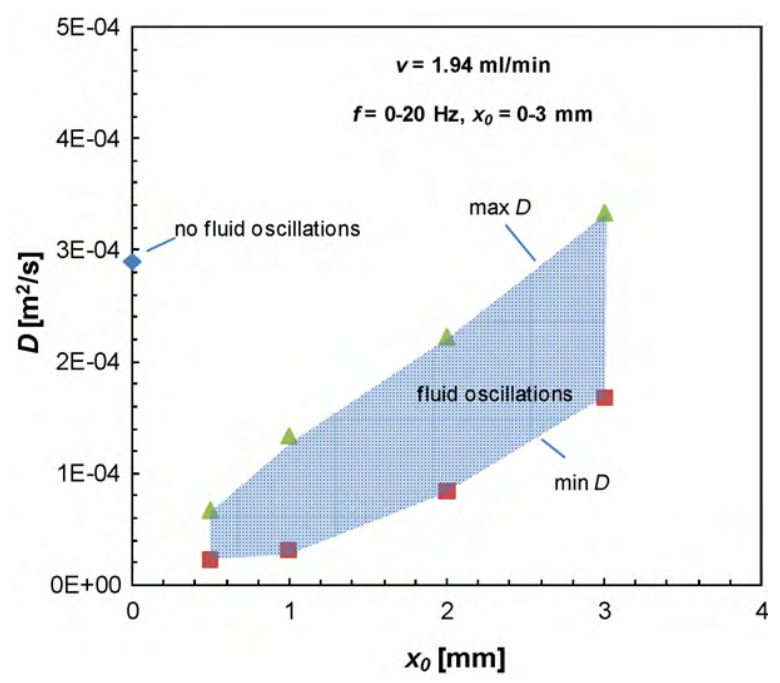

Fig. 11. Limits of axial dispersion coefficient, $D\left(\mathrm{~m}^{2} / \mathrm{s}\right)$ achievable with the introduction of fluid oscillations, as determined from Fig. 10a $(v=1.94 \mathrm{ml} / \mathrm{min})$.

The hydrodynamic model parameters were in general very sensitive to the method selected to best-fit the experimental results and the analytical equations. This was especially important for the data obtained at steady flow conditions, which resulted in broad tracer responses from the tube. The convective laminar flow [26] observed at $v=1.94 \mathrm{ml} / \mathrm{min}$ as above presented in Fig. 5a resulted in an $E_{\theta}$-diagram with a asymmetric peak at $\theta=0.5$ and a tail that extends beyond $\theta=3$. Such type of response could not be accurately predicted by any of the hydrodynamic models herein considered because of the significant radial concentration gradients presented by the parabolic laminar profile within the tube. The moment comparison is often chosen by many authors to estimate the backmixing for being of easy computation from numerical integration. Nevertheless, major drawbacks limit the applicability of moments technique to the modelling of flow systems, such as: (i) the lack of knowledge of the quality of the fit of the model and (ii) emphasis given by this numerical method to the data in the tail of $C$-curves, which is usually less accurate [14].

Parameters for the remaining models were estimated following the same methods and, for simplicity, they were herein represented as a function of $D$. Fig. 12 shows the plots $G$ versus $D$ and $N_{t i s}$ versus $D$ as estimated by method II (transfer function match) and III ( $E_{\theta}$-diagram match). In general, the parameter estimated by direct comparison of transfer functions in the Laplace domain (method II) returned the most consistent results as can be seen in Table 3, therefore the discussion below was based only on the data obtained through method II.

The tanks-in-series with backflow (with corresponding parameter $G$ ) and the plug flow with axial dispersion (with parameter $D$ ) models independently fitted to the experimental data have returned an excellent cross-correlation; $D$ and $G$ for a system presenting closed-closed boundaries system are related by the equation $G=N_{s w} \times D / u L-0.5$, where $N_{s w}$ is the number of tanksin-series (stages) considered (i.e. $N_{s w}=26$ ) [24].

The tanks-in-series model without backflow (model D) returned also consistent results, nevertheless it was unable to match reasonably the tracer response curves in the tube at the high values of $x_{0}$ herein tested. This was because high values of $x_{0}$ generated extended backflow rates that are not physically considered in the mass balance to the hydrodynamic model. For small values of $x_{0}$, the fitting was generally good which demonstrates that each cavity can actually behave as a small, individual CSTR in the constricted tube. 

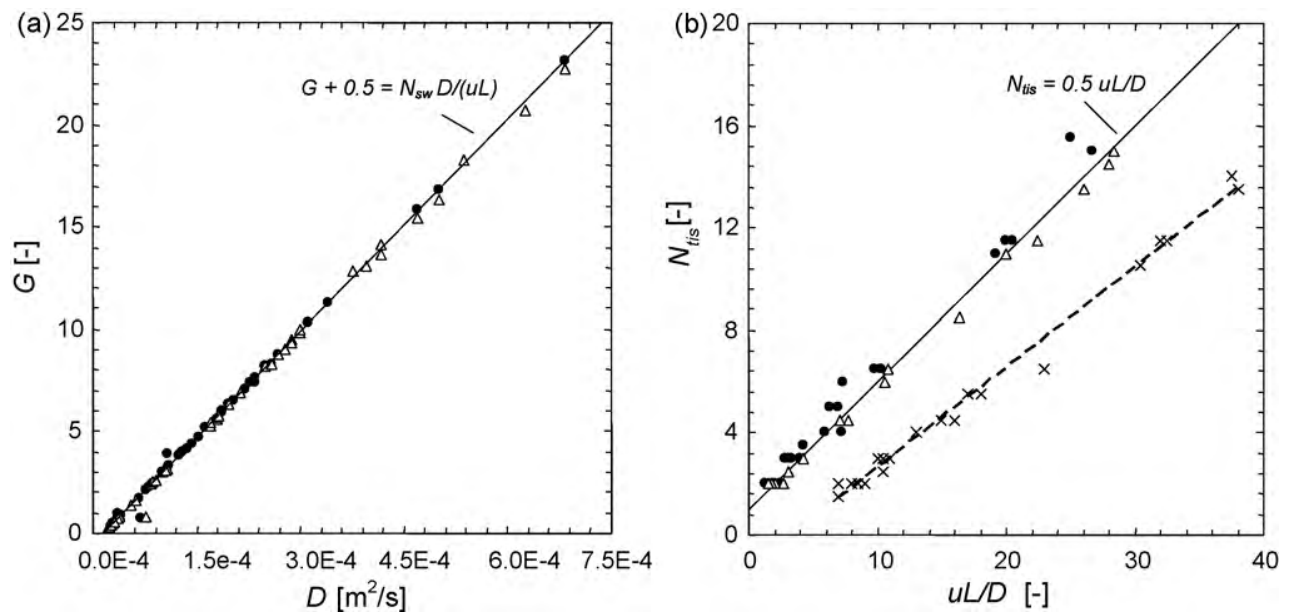

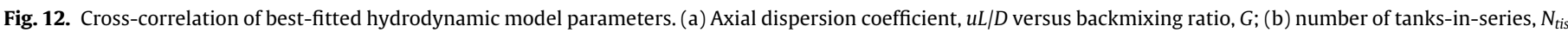

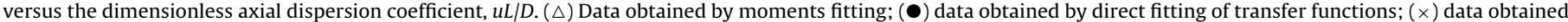
by direct fitting of $F_{\theta}$-diagrams [26]. Line represent observed or theoretical trend; for best-fitted equations and cross-correlation coefficients see Table 3 .

The fitting procedure based on the transfer functions as above shown was concluded to be the most simple and effective process to extract the model parameters from the hydrodynamic models. The applicability of the time-domain fitting technique is limited by the small number of analytical solutions that are possible to obtain from the differential equations that represent the mass balances in the hydrodynamic systems. One exception is the axial dispersion model with open-open boundary conditions and through-the-wall measurements as presented by Levenspiel [22]. In the flow system herein studied, it was found that when the experimental data was fitted with Levenspiel's equation the values of $D$ were about 30-35\% underestimated as shown by the second data set in Fig. 12b.

In summary, the RTD in the meso-tube studied with different hydrodynamic models and model parameters estimated through different numerical techniques has clearly demonstrated the possibility of controlling the extent pf liquid backmixing in the through the use of oscillatory flow mixing.

\subsection{Relevance of this platform to process intensification}

The development of continuous platforms delivering a controllable RTD is fundamental for the design of intensified, green chemical or biological processes. Previous work with the same platform herein studied have demonstrated its capacity to handle multiphase flow processing, with improved performances in terms of particles suspension or enhanced contacting between immiscible phases (gas, liquid or solid) (see for example Reis et al. [1]).

The control of liquid backmixing in process intensification is as important as promoting the enhanced contact between immiscible phases; the former controls the conversion rates and selectivities in reactive or separation processes. The level of fine-tuning of RTDs shown by this meso-scale platform would be relevant to the process intensification of added-value pharmaceutical products as it can support, e.g. higher conversions in chemical reactions in a continuous fashion, when compared to the conventional CSTR. To better illustrate the impact of RTD response on the overall reactor performance, the experimental curves shown in the text above were used to predict the conversion for a first-order, homogenous reactions in the tube.

A distribution function can be directly applied to predict chemical reactor behaviour as follows [24]:

$\bar{C}_{A}=\int_{0}^{\infty} C_{A}(\theta) E_{\theta} d \theta$

For a first-order reaction it becomes:

$1-\bar{X}=\frac{\bar{C}_{A}}{C_{A_{\text {in }}}}=\int_{0}^{\infty} e^{-k \theta} E_{\theta} d \theta$

The above integral is in fact the definition of Laplace transform with respect to $\theta$ of $E_{\theta}$, but with $k$ substituted by $T$ (Laplace operator). Thus, the conversion in a first-order reaction can be found from the transforms of any $E_{\theta}$ by merely replacing $k$ for $T$, as shown elsewhere [14].

Fig. 13 summarises the conversion, $\bar{X}$ estimated from the response curves for a first-order isothermal reaction running in the periodically constricted tube in four different flow conditions: (1) steady laminar flow (i.e. $R e_{0}=0$ ) at $v=1.94 \mathrm{ml} / \mathrm{min}$; (2) oscillatory flow at $x_{0}=0.5 \mathrm{~mm}$ and $f=15 \mathrm{~Hz}$; (3) oscillatory flow, with $x_{0}=1 \mathrm{~mm}$ and $f=10 \mathrm{~Hz}$; (4) oscillatory flow at $x_{0}=3 \mathrm{~mm}$ and $f=20 \mathrm{~Hz}$ (i.e. maximum liquid backmixing scenario). Conversions were also compared to a completely segregated flow (CSTR) and ideal tubular flow (i.e. a PFR). The use of oscillatory flow in this platform can help deliver conversions close to those only achievable with an ideal PFR.

Table 4 summarises the estimated deviations in conversion from a PFR for a reference value of $k \bar{t}=4$ as estimated from Fig. 13. Deviations in $\bar{X}$ in the smooth-periodically constricted tube were as low as $1.2 \%$ in relation to the $\bar{X}$ expected for an ideal PFR. The use of strong fluid oscillations, namely $f=20 \mathrm{~Hz}$ and $x_{0}=3 \mathrm{~mm}$, delivering an extended liquid backmixing as above shown, would result in a

Table 3

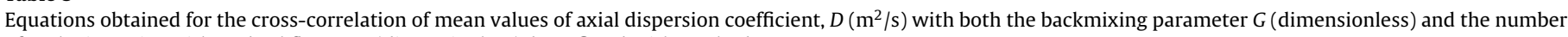
of tanks-in-series without backflow, $N_{\text {tis }}$ (dimensionless), best-fitted with methods I-III.

\begin{tabular}{|c|c|c|}
\hline Best-fitting method & $G$ versus $D$ & $N_{\text {tis }}$ versus $D$ \\
\hline I. Moments fitting & $G=22.84 u L / D+0.23, r=0.9975$ & $N_{t i s}=0.49 u L / D+0.90, r=0.9976$ \\
\hline II. Direct fitting of transfer function, $g(T)$ & $G=26.06 u L / D-0.55, r=0.9993$ & $N_{t i s}=0.53 u L / D+1.17, r=0.9881$ \\
\hline III. Direct fitting of $F_{\theta}$-diagram & - & $N_{t i s}=0.39 u L / D-1.28, r=0.9913$ \\
\hline Observed and theoretical trend & $G \approx 26 u L / D-0.5$ from $[23]$ & $N_{t i s} \approx 0.5 u L / D+1$ from $[21]$ \\
\hline
\end{tabular}


Table 4

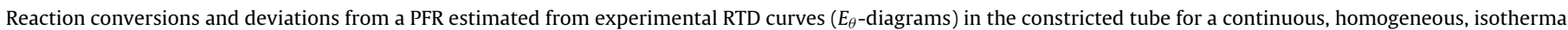
first-order reaction at $v=1.94 \mathrm{ml} / \mathrm{min}$.

\begin{tabular}{|c|c|c|c|c|c|c|}
\hline Per cent conversion for & CSTR & $3 \mathrm{~mm}, 20 \mathrm{~Hz}$ & $R e_{0}=0$ & $0.5 \mathrm{~mm}, 15 \mathrm{~Hz}$ & $1 \mathrm{~mm}, 10 \mathrm{~Hz}$ & PFR \\
\hline Predicted conversion & 80.0 & 92.0 & 94.4 & 96.6 & 97.0 & 98.2 \\
\hline Difference in conversion & -18.2 & -6.2 & -3.8 & -1.6 & -1.2 & 0 \\
\hline
\end{tabular}

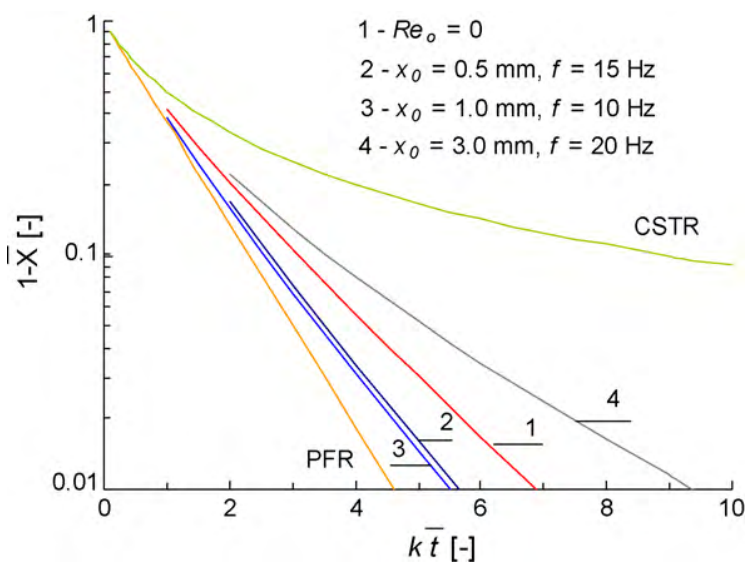

Fig. 13. Reaction conversions, $\bar{X}$ predicted for a continuous homogeneous, isothermal chemical reaction in the constricted at steady and oscillatory flow regimes $(v=1.94 \mathrm{ml} / \mathrm{min})$.

deviation of $\bar{X}$ as low as $6.2 \%$, which remains significantly smaller than the $18.2 \%$ reduction in $\bar{X}$ estimated for a completely segregated flow in a CSTR.

Industrially, a near plug flow can be obtained by flowing the solutions/reagents though long tubes at high $R e_{n}$ numbers, in the order 10,000 (i.e. highly turbulent flow). This is particularly important to keep solids in suspension and minimise fluid segregation, as is the case of (bio)catalytic reactions. However, to operate in the turbulent flow regime, large volumes of reagents have to be pumped through the tubular system per unit of time, which represents highenergy consumptions and can only deliver residence times in the order of few seconds, which is not compatible with the production of most of fine chemicals and added-value pharmaceutical products. Nevertheless, it was shown in this study that a platform based on smooth-periodic constrictions in a small-diameter tube geometry and oscillatory flow mixing can deliver sharp residence times in laminar flow with residence times of minutes to hours, allowing the sustainable kilogram-per-day production of added-value products.

\section{Conclusions}

Liquid backmixing was studied for a $4.5 \mathrm{~mm}$ internal diameter tube presenting smooth-periodic constrictions and operating with oscillatory flow mixing (OFM) at laminar flow conditions.

Distribution functions monitored with optical micro-probes following a step injection of a dye tracer at the inlet have shown that the extent of liquid backmixing in the constricted meso-tube can be fine-tuned according to the fluid oscillations conditions in the input. The use of OFM resulted in at least one-order of magnitude reduction in the liquid backmixing in comparison with the steady flow situation. Both $f$ and $x_{0}$ appear controlling the liquid backmixing in the tube, nevertheless values of $x_{0} \leq 1 \mathrm{~mm}$ and $f=10 \mathrm{~Hz}$ are preferable for situations where a minimum deviation from plug flow is desirable.

The sharp residence time distributions were successfully modelled using three hydrodynamic models: (i) tanks-in-series, (ii) tanks-in-series with backflow and (iii) plug flow with axial dispersion; however, the tanks-in-series model failed representing the

liquid backmixing at higher values of $f$ and $x_{0}$.

This platform is relevant for supporting high selectivity and conversions for reactive flows as exemplified for a first-order isothermic reaction. The use of laminar net flow rates and the capability of handling continuous multiphase flows suggest the new platform is relevant to the process intensification of upstream or downstream production of added-value chemical and pharmaceutical products.

\section{Acknowledgments}

NR is grateful to Fundação para a Ciência e a Tecnologia, FCT, Portugal for financial support.

\section{Appendix A. Nomenclature}

$I$

$d$

$d_{0} \quad$ free tube-internal diameter in the constriction ( $\mathrm{m}$ )

$\bar{d} \quad$ mean internal diameter of the tube $(\mathrm{m})$

D axial dispersion coefficient $\left(\mathrm{m}^{2} / \mathrm{s}\right)$

E normalised tracer concentration, dimensionless

$f \quad$ fluid oscillation frequency $(\mathrm{Hz})$

$F \quad$ cumulative tracer concentration, dimensionless

$g(T) \quad$ transfer function, dimensionless

$G \quad$ backmixing ratio, dimensionless

$k \quad$ first-order kinetic constant (1/s)

$l \quad$ inter-constrictions distance $(\mathrm{m})$

$N_{s w} \quad$ total number of stages, dimensionless

$N_{\text {tis }} \quad$ number of tanks-in-series, dimensionless

$r \quad$ cross-correlation coefficient, dimensionless

$\operatorname{Re}_{n} \quad$ net flow Reynolds number, $\operatorname{Re}_{n}=u_{L S} \rho d / \mu$

$R e_{0} \quad$ oscillatory Reynolds number, $R e_{0}=2 \pi f x_{0} \rho d / \mu$

$t$ time (s)

$\bar{t} \quad$ mean residence time of the tracer (s)

$T \quad$ Laplace operator, dimensionless

$u_{L S} \quad$ superficial liquid velocity $(\mathrm{mm} / \mathrm{s})$

$v \quad$ volumetric flow rate $(\mathrm{ml} / \mathrm{min})$

$V \quad$ internal volume $(\mathrm{ml})$

$x \quad$ tracer concentration $(\mathrm{g} / \mathrm{l})$

$\bar{x} \quad$ Laplace's transform of the pulse response, dimensionless

$x_{0} \quad$ fluid oscillation amplitude ( $\mathrm{mm}$ )

$\bar{X} \quad$ conversion, dimensionless

$L \quad$ tube length $(\mathrm{m})$

$z \quad$ dimensionless distance

\section{Subscripts/superscripts}

in $\quad$ incoming $(x)$

out outgoing $(x)$

$t$ time

$\theta \quad$ dimensionless time

\section{Greek letters}

$\alpha \quad$ cross-free section area, dimensionless

$\delta \quad$ constriction length $(\mathrm{mm})$

$\lambda \quad$ wavelength ( $\mathrm{nm})$

$\mu \quad$ fluid viscosity $(\mathrm{kg} /(\mathrm{m} \mathrm{s}))$ 
$\rho \quad$ density of fluid $(\mathrm{g} / \mathrm{l})$

$\sigma^{2} \quad$ variance $\left(\mathrm{s}^{2}\right)$

$\tau \quad$ mean hydraulic time, $t=V / v$

\section{References}

[1] N. Reis, A.P. Harvey, A.A. Vicente, J.A. Teixeira, M.R. Mackley, Fluid mechanics and design aspects of a novel oscillatory flow meso-reactor, Chemical Engineering Research \& Design 83 (A4) (2005) 357-371.

[2] N. Reis, P.C. Mena, A.A. Vicente, J.A. Teixeira, F.A. Rocha, The intensification of gas-liquid flows with a periodic, constricted oscillatory-meso tube, Chemical Engineering Science 62 (24) (2007) 7454-7462.

[3] N Reis, C.N. Gonçalves, A.A. Vicente, J.A. Teixeira, Proof-of-concept of a novel micro-bioreactor for fast development of industrial bioprocesses, Biotechnology and Bioengineering 95 (4) (2006) 744-753.

[4] M.Zheng, M.R. Mackley, The axial dispersion performance of an oscillatory flow meso-reactor with relevance to continuous flow operation, Chemical Engineering Science 63 (7) (2008) 1788-1799.

[5] N. Reis, A.A. Vicente, J.A. Teixeira, M.R. Mackley, Residence times and mixing of a novel continuous oscillatory flow screening reactor, Chemical Engineering Science 59 (22-23) (2004) 4967-4974.

[6] K.D.P. Nigam, A.K. Saxena, Residence time distribution in straight and curved tubes, in: N.P. Chermisinoff (Ed.), Encyclopedia of Fluid Mechanics, vol. 1, Gulf Publishing Co., Morganville, TX, 1986, pp. 675-762.

[7] S. Vashisth, V. Kumar, K.D.P. Nigam, A review on the potential applications of curved geometries in process industry, Industrial Engineering and Chemical Research 47 (10) (2008) 3291-3337.

[8] W. Lu, S. Hwang, C. Chang, Liquid mixing in internal loop airlift reactors, Industrial Engineering and Chemical Research 33 (1994) 2180-2186.

[9] R.T. Hatch, Experimental and theoretical studies of oxygen transfer in the airlift reactor, Ph.D. thesis, Massachusetts Institute of Technology, Massachusetts, 1973.

[10] A.S. Andersse, E.T. White, Parameter estimation by weighted moments method, Chemical Engineering Science 26 (8) (1971) 1203.

[11] S.K. Gangwal, R.R. Hudgins, A.W. Bryson, P.L. Silvesto, Interpretation of chromatographic peaks by Fourier analysis, Canadian Journal of Chemical Engineering 49 (1) (1971) 113.
[12] P. Verlaan, A.M.M. Vaneijs, J. Tramper, K. Vantriet, K.C.A.M. Luyben, Estimation of axial-dispersion in individual-sections of an airlift-loop reactor, Chemical Engineering Science 44 (5) (1989) 1139-1146.

[13] P.L. Mills, M.P. Dudukovic, Convolution and deconvolution of nonideal tracer response data with application to 3 -phase packed-beds, Computers \& Chemical Engineering 13 (8) (1989) 881-898.

[14] G.F. Froment, K.B. Bischoff, Chemical Reactor Analysis and Design, John Wiley \& Sons, New York, 1990.

[15] M.R. Mackley, X. Ni, Mixing and dispersion in a baffled tube for steady laminar and pulsatile flow, Chemical Engineering Science 46 (12) (1991) 31393151.

[16] M.R. Mackley, X. Ni, Experimental fluid dispersion measurements in periodic baffled tube arrays, Chemical Engineering Science 48 (18) (1993) 32933305.

[17] X.W. Ni, N.E. Pereira, Parameters affecting fluid dispersion in a continuous oscillatory baffled tube, AIChE Journal 46 (1) (2000) 37-45.

[18] X.W. Ni, S.W. Gao, D.W. Pritchard, Study of mass-transfer in yeast in a pulsed baffled bioreactor, Biotechnology and Bioengineering 45 (2) (1995) 165175.

[19] X.W. Ni, Y.S. de Gelicourt, J. Neil, T. Howes, On the effect of tracer density on axial dispersion in a batch oscillatory baffled column, Chemical Engineering Journal 85 (1) (2002) 17-25.

[20] A.W. Fitch, X. Ni, On the determination of axial dispersion coefficient in a batch oscillatory baffled column using laser induced fluorescence, Chemica Engineering Journal $92(1-3)(2003)$ 243-253.

[21] C.L. Briens, A. Margaritis, G. Wild, A new stochastic-model and measurement errors in residence time distributions of multiphase reactors, Chemical Engineering Science 50 (2) (1995) 279-287.

[22] E.B. Nauman, B.A. Buffham, Mixing in Continuous Flow Systems, Wiley Intersciences, New York, 1983

[23] C.F. Wen, L.T. Fan, Models for Flow Systems and Chemical Reactors, Marcel Dekker, New York, 1975

[24] K.R. Westerterp, W.P.M. van Swaaij, A.A.C.M. Beenackers, Chemical Reactor Design and Operation, John Wiley \& Sons, New York, 1963.

[25] J.C. Mecklenburgh, S. Hartland, The Theory of Backmixing, John Wiley \& Sons Inc., New York, 1976.

[26] O. Levenspiel, Chemical Reaction Engineering, John Wiley \& Sons Inc., New York, 1972.

[27] P.V. Danckwerts, Continuous flow systems-distribution of residence times, Chemical Engineering Science 2 (1) (1953) 1-13. 